\title{
Experimental QCD results and impact on LHC physics
}

\author{
Emmanuel Sauvan* \\ Laboratoire d'Annecy-le-vieux de Physique des Particules (LAPP), CNRS/IN2P3 et Université \\ de Savoie, \\ Centre de Physique des Particules de Marseille (CPPM) \\ E-mail: sauvan@lapp.in2p3.fr
}

\begin{abstract}
Recent experimental results on the understanding of the QCD structure of the proton are presented. A particular emphasis is given on the gluon content of the proton, whose precise knowledge has direct implications for theoretical predictions at the LHC. The most important data used in the determination of the parton density functions (PDFs) are ep Deep Inelastic Scattering (DIS) cross sections measured at HERA. New combined data from H1 and ZEUS experiments are improving further the experimental accuracy, in particular at low Bjorken $x$, and lead to precise predictions of cross sections for LHC phenomena. The flavour content of the proton is further studied using final states with charm and beauty in DIS $e p$ and $p \bar{p}$ collisions. Finally, diffractive reactions involving a hard scale are mentioned as a tool for investigating the low $x$ structure of the proton.
\end{abstract}

35th International Conference of High Energy Physics

July 22-28, 2010

Paris, France

${ }^{*}$ Speaker. 


\section{Introduction}

The understanding of high energy collisions involving protons relies on the factorisation theorem, which states that the cross section is a convolution of a perturbative part, which describes the parton-parton interactions at high energy, with a non-perturbative part that describes the momentum distributions of the partons inside the proton [1]. The parton distribution functions $f\left(x, Q^{2}\right)$ (PDFs) encapsulate the parton content of the proton. They depend on the proton momentum fraction carried by the interacting parton, $x$, and the scale $Q^{2}$ at which the proton is probed. Their form cannot yet be calculated within QCD. Nevertheless, their evolution with $Q^{2}$ is predicted in perturbative QCD by the DGLAP equations [2]. Therefore, the PDFs need only to be parametrised as a function of $x$ at a given $Q^{2}$ scale. The status of the proton structure investigations can be represented in the kinematic plane $x-Q^{2}$, displaying regions accessed by fixed target experiments and high energy colliders, as in figure 1(a). The large and unique kinematic coverage of HERA can be observed, allowing to probe the proton structure in the perturbative domain down to low $x$ values of $\sim 10^{-5}$. This regime is particularly important for LHC physics since the production of particles of moderate masses like $W, Z$ or Higgs bosons will involve mainly partons of medium to low $x$ and are therefore dominated by the scattering of gluons and sea quarks. A precise knowledge of the proton gluon density is thus an important input for the calculation of main SM processes at the LHC. The measurements are complemented by the $p \bar{p}$ collider Tevatron, where $W$ boson and jet production processes bring additional constraints to the proton structure at high $x$, a regime which correspond to the production of possible new massive particles at the LHC. The parametrisation in $x$ of the PDFs is constrained by fits to the different available measurements, from $e p$ DIS in fixed target experiments and collisions at HERA and from $p \bar{p}$ collisions at the Tevatron. Different group of PDF sets exist, with differences in the choice of the data sets used in the fit, as well as in the treatment of theoretical inputs. Variations between the predictions of different PDFs sets, which are sometimes larger than the quoted uncertainties, raise the question of the theoretical errors associated to the extracted PDFs and of the present accuracy of our knowledge of the proton structure. For example, differences in the gluon density function leads to different gluon-gluon luminosities predicted for the LHC, as represented in figure 1. These differences between PDFs sets also translate into spreads of $\sim 7 \%$ for the predicted Higgs production cross section and of the order of 3 to $5 \%$ for $W$ and $Z$ production. A good remedy to understand and reduce these differences is to input new data, in order to better constraint the PDFs and possible theoretical treatments.

\section{The spine of PDFs determination}

Precise PDFs down to very small $x \approx 0.0001$ can be determined from HERA inclusive neutral current (NC) and charged current (CC) DIS data alone. Recently, H1 and ZEUS Collaborations combined the data collected during the HERA I phase (1994-2000) [4]. The average procedure is based on the minimisation of a $\chi^{2}$ function, whose free parameters are not only the average values in each measurement point but also the correlated systematics shifts from each data set. This leads to an effective cross-calibration of the two experiments, greatly reducing systematic errors. A new combination including high $Q^{2} \mathrm{NC}$ and $\mathrm{CC}$ data from the HERA II phase has been released by $\mathrm{H} 1$ and ZEUS for this conference [5]. The inclusion of the large HERA II data sets leads to a reduced 

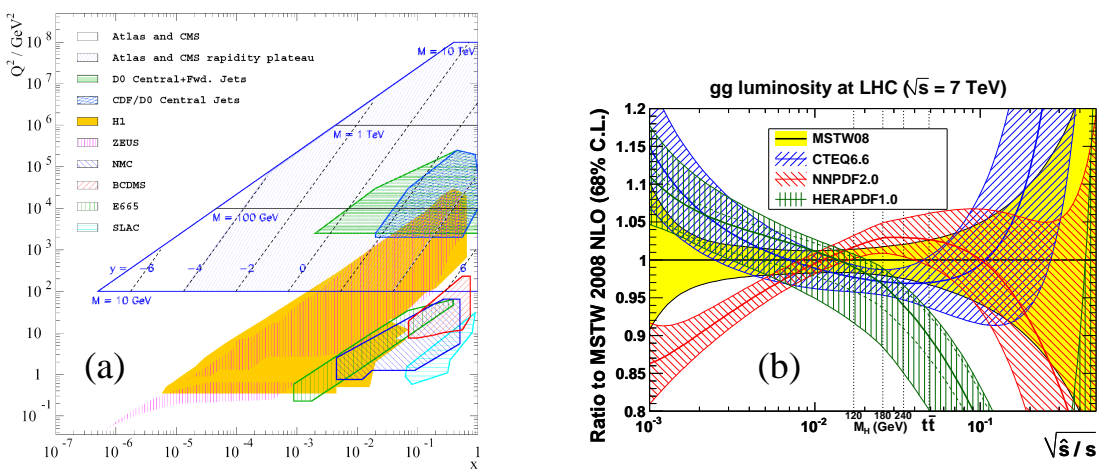

Figure 1: (a): The kinematic plane $x-Q^{2}$ with the regions covered by fixed target experiments, HERA and Tevatron colliders. The domain probed at LHC for $14 \mathrm{TeV}$ collisions is also indicated. (b): $g-g$ luminosity for $7 \mathrm{TeV}$ LHC collisions, predicted by different PDFs sets (from [3]).
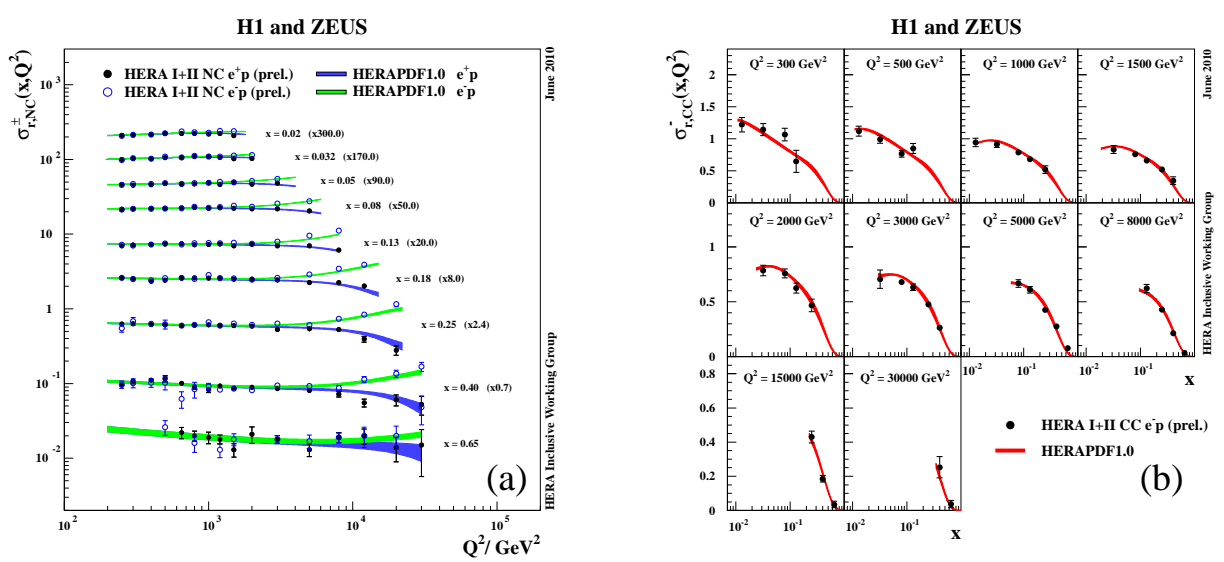

Figure 2: (a): HERA I+II combined NC $e^{+} p$ and $e^{-} p$ reduced cross section. (b): HERA I+II combined CC $e^{-} p$ reduced cross section. The measurements are compared with the HERAPDF1.0 fit.

statistic uncertainty, especially for $e^{-} p$ data, as seen in figure 2, and brings further constraints on proton valence quark densities and on the separation of $u$ and $d$ quark densities from HERA data alone. These new data have been used to perform a new stand alone NLO QCD fit using the same procedure as used for HERAPDF1.0 [6]. The resulting PDFs extrapolated at a scale of $Q^{2}=10000 \mathrm{GeV}^{2}$ are presented in figure $3(\mathrm{~b})$. We can observe that the precision of the PDFs at high- $x$ is considerably improved, particularly in the valence sector.

\section{Gluons of high and medium $x$ in the proton}

Jet production at HERA proceeds via the boson-gluon fusion (BGF) and QCD Compton processes. It therefore provides a direct access to the gluon density via the BGF process and is also sensitive to $\alpha_{s}$ and quark densities. New measurements of differential inclusive jet, di-jet and tri-jet cross sections in DIS and photoproduction have been performed by the ZEUS experiment using HERA II data with more than a three-fold increase in statistics compared to previous analyses $[9,10,11]$. These measurements represent a stringent test of perturbative QCD, NLO calculations providing a good description of the data. When included in PDFs fits, the HERA jet data 

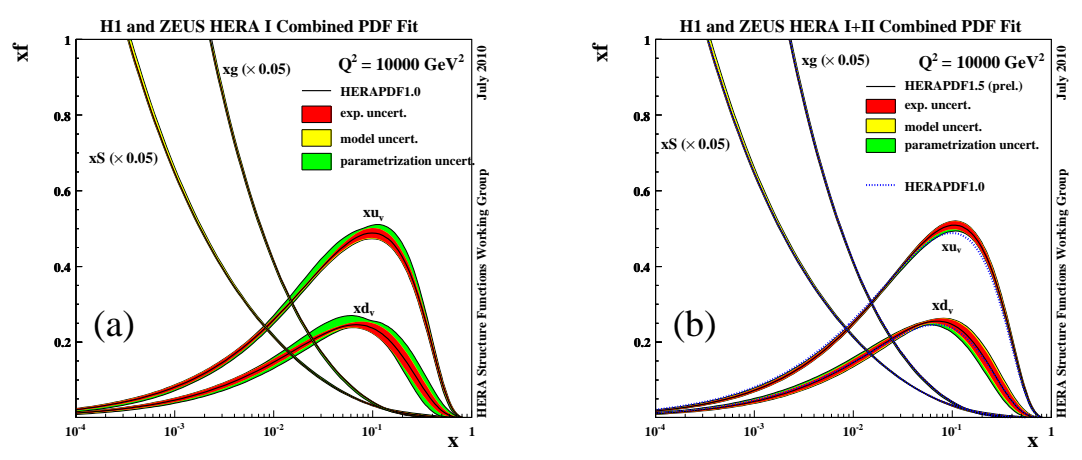

Figure 3: The parton distribution functions from HERAPDF1.0 (a) and HERAPDF1.5 (b) $x u_{v}, x d_{v}, x S=$ $2 x(\bar{U}+\bar{D})$ and $x g$, at $Q^{2}=10000 \mathrm{GeV}^{2}$.
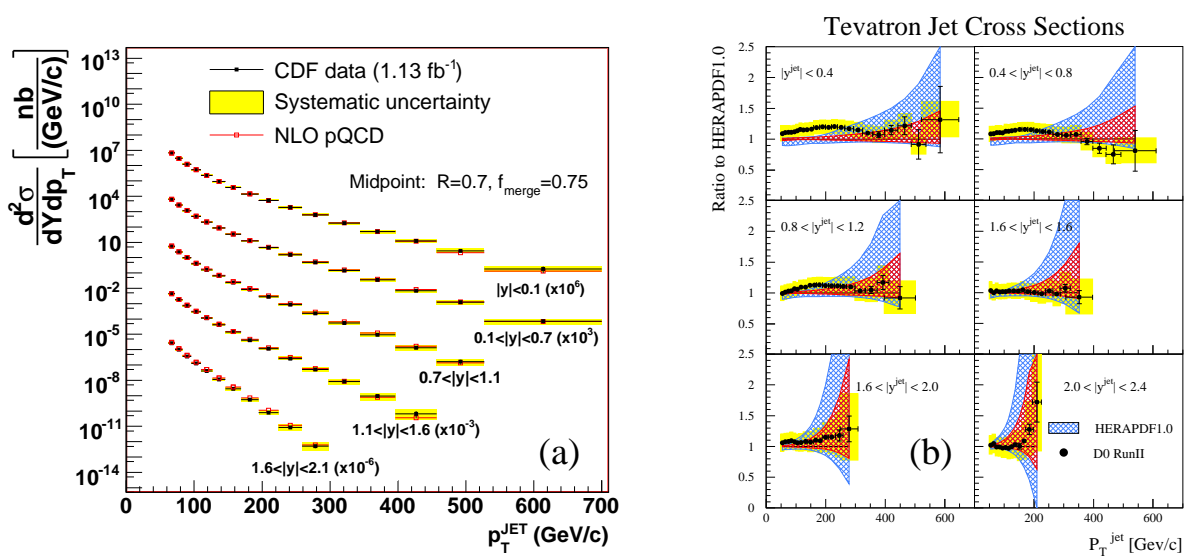

Figure 4: (a): Inclusive jet cross sections measured by the CDF experiment. (b): Ratio of high $E_{T}$ jet cross sections measured by DØ to HERAPDF1.0 prediction. The systematic experimental error is indicated in yellow, the total uncertainty in blue and the experimental one in red (from [14]).

will be mainly useful to further constrain the proton gluon density for proton momentum fractions in the range $0.01<x<0.4$, which is of high relevance for many LHC processes in the central rapidity range of ATLAS and CMS detectors such as $g g \rightarrow H$. At the Tevatron, the production of highly energetic jets provides constraints on the proton gluon density up to the largest $x$ close to unity. Recent measurements of the inclusive jet production have been performed by both the CDF and DØ Collaborations, using a large statistics sample of run II data [12, 13]. Measured cross sections are in good agreement with the NLO QCD prediction, as can be seen in figure 4(a) for CDF measurements. The accuracy of the data has been greatly improved and the dominant experimental uncertainty related to the jet energy scale has been reduced to $1 \%$. The main experimental uncertainties originate from the PDFs and exceed the experimental precision, as exemplified in figure 4(b). These Tevatron Run II jet data are therefore now used in global PDF fits, replacing Tevatron Run I data $[15,16]$. The impact is a smaller high- $x$ proton gluon density preferred by these data. But, due to a large number of sizable correlated systematic uncertainties in these jet data, their inclusion in global PDF fits leads to only a small reduction of the fractional uncertainty on the high- $x$ proton gluon density [15]. 


\section{Gluons and heavy flavours in the proton}

The longitudinal structure function $F_{L}$ is a fundamental form factor of the proton which is directly related to the gluon density in the proton $[18,19]$. At low $x, F_{L}$ is a direct measure of the gluon distribution to a good approximation. A direct measurement of $F_{L}$ can be performed if the cross section $\sigma\left(E_{p}\right) \sim F_{2}\left(x, Q^{2}\right)+f(y) F_{L}\left(x, Q^{2}\right)$ is measured at fixed $x$ and $Q^{2}$ but variable $y$. This was achieved at the end of the HERA running by varying the collision energy $\sqrt{s}$ in two dedicated runs at lower proton beam energies of 460 and $575 \mathrm{GeV}$. Data from the H1 and ZEUS experiments have been combined to provide a direct measurement of $F_{L}$ in the low $x$ regime and for $Q^{2}$ down to $2.5 \mathrm{GeV}^{2}$. The measurements averaged in $x$ for each $Q^{2}$ bins are shown in figure 5(a). The lowest $x$ and $Q^{2}$ measurements exhibit an interesting sensitivity to differences in theoretical treatment in PDFs fits. The predictions at NNLO seem to return a better description of the $F_{L}$ data compared to HERAPDF1.0 fit performed at NLO. Heavy quark flavours in the proton being radiatively generated from gluons, different theoretical treatments of heavy flavours in PDF fits lead to different gluon densities and therefore to different predictions for $F_{L}$, as exemplified in figure 5(b).

The understanding of the heavy flavour content of the proton is of particular importance for searches of particle that directly couple to the mass at the LHC, especially for the Higgs studies. In DIS, interactions initiated by heavy quarks can be tagged by the presence of heavy flavours signals in the final state. Such interactions occur when gluons in the proton fluctuate to a pair of heavy quarks, one of which interacting with the photon (boson-gluon fusion process). Therefore, the study of heavy flavour interactions in DIS may also constrain the gluon content of the proton. Charm events are tagged at HERA using fully reconstructed $D$ mesons or displaced track impact parameters. Several results from H1 and ZEUS experiments on the measurement of the charm contribution $F_{2}^{c \bar{c}}$ to the proton structure function $F_{2}$ have been recently combined [21]. The averaged $F_{2}^{c \bar{c}}$ obtained is presented in figure 6(a). The precision of the averaged data is comparable or better than the difference among the various predictions and will therefore lead to improvements in the proton structure determination. A recent analysis has shown that the inclusion of these averaged $F_{2}^{c \bar{c}}$ data helps to reduce the uncertainties due to the heavy flavour treatment in PDF fits on the $W^{ \pm}$and $Z$ production cross section at LHC to below $1 \%$ [22]. Beauty production at HERA probes the gluon density mainly at larger proton momentum fractions $x \sim 0.01$, where it is well known from scaling violations. The available measurements of the structure function $F_{2}^{b \bar{b}}$ at HERA are presented in the figure 6(b). This includes new measurements from the ZEUS Collaboration using secondary vertex tagging or electron decay of beauty hadrons and exploiting the full HERA II data set [23], leading to a significant reduction of the statistical uncertainties. The full potential of these data will be obtained by the combination of $\mathrm{H} 1$ and ZEUS measurements and may provide in a near future constraints on the effective beauty quark density in the proton, which can then be used to improve predictions at the LHC for processes such as $b \bar{b} \rightarrow H$ or $b \bar{b} \rightarrow Z$.

The heavy quark content of the proton can also be tested at the Tevatron using measurements of the associated production of a boson $(V)$ and an heavy quark $(Q)$. Such events are produced primarily through the QCD Compton-like scattering process $\gamma Q \rightarrow V Q$, but also through quarkantiquark annihilation $q \bar{q} \rightarrow V \gamma \rightarrow V Q \bar{Q}$. Such processes could therefore provide some sensitivity to gluons and heavy quarks in the proton and their measurements provide good tests of perturbative 

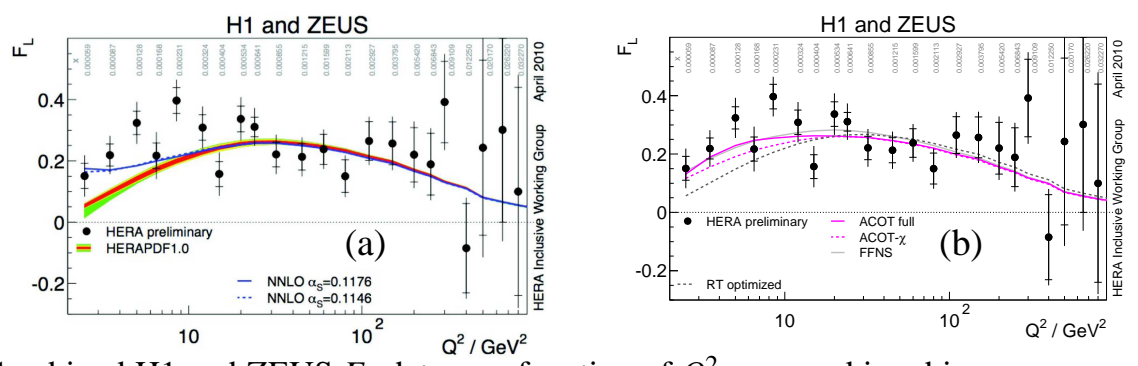

Figure 5: Combined $\mathrm{H} 1$ and ZEUS $F_{L}$ data as a function of $Q^{2}$ averaged in $x$ bins versus predictions from HERAPDF1.0 performed at NLO and NNLO (a) or performed under various heavy flavour treatments (b).
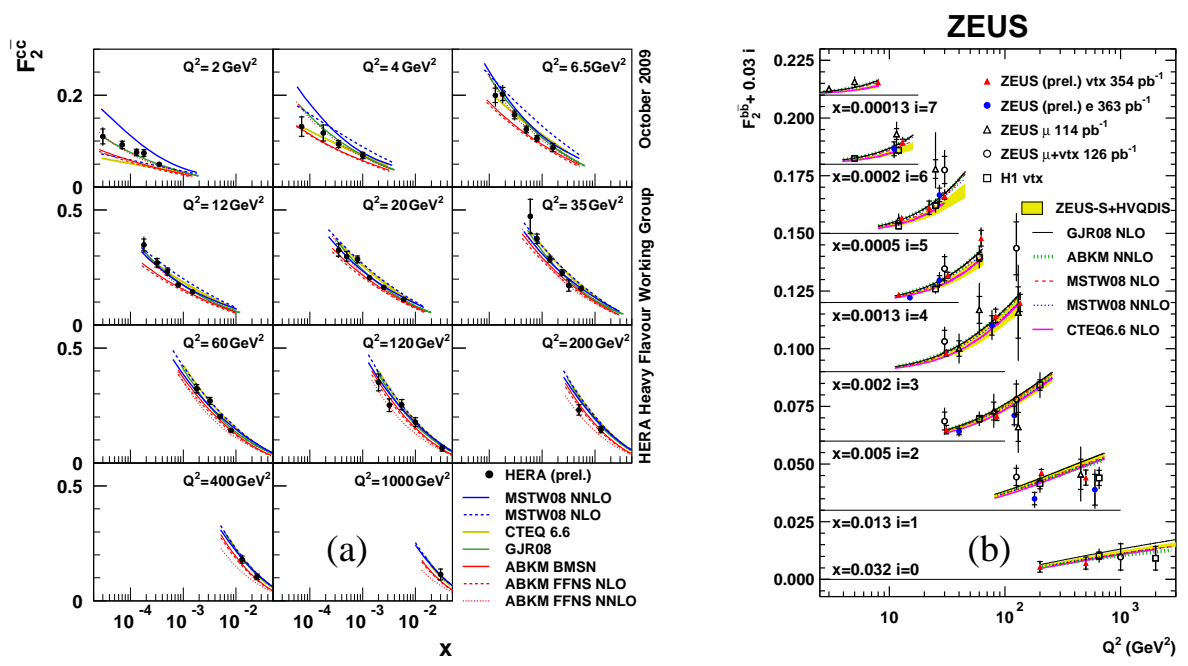

Figure 6: HERA averaged $F_{2}^{c \bar{c}}$ (a) compared to recent QCD predictions and $F_{2}^{b \bar{b}}$ (b) as a function of $Q^{2}$ for various $x$ values measured at HERA, compared to different theoretical predictions.

QCD calculations. They are also important background in different searches and need thus to be controlled. Differential cross sections for the inclusive production of a photon in association with a $c$ or $b$ jet in $p \bar{p}$ collisions have been measured by the D $\emptyset$ Collaboration [24]. These measurements probe the proton gluon and $b, c$ quark PDFs in the range $0.01 \lesssim x \lesssim 0.3$. The pQCD prediction agrees with the measured cross section for $\gamma+b+X$ production, but the experimental error is still larger than the PDF uncertainty. For $\gamma+c+X$ production, the measured cross section is higher than the prediction for $P_{T}^{\gamma}>70 \mathrm{GeV}$. Concerning the production of a $Z$ boson with a $b$-jet, first differential cross sections have been measured by the CDF experiment [25]. Large variations are seen between the theoretical predictions as no full NLO QCD calculation is available for this process. The predictions generally describe the data, but the agreement is better for those predictions that use a low value for the renormalisation and factorisation scales.

\section{Another view on the gluon at small $x$ : hard diffraction}

At low $x$ values, the gluon content of the proton may also be probed by hard diffractive interactions. Such interactions result from a colorless exchange and can be viewed at leading order in pQCD as a two-gluon exchange. Experimentally, diffractive interactions are characterised by a final 


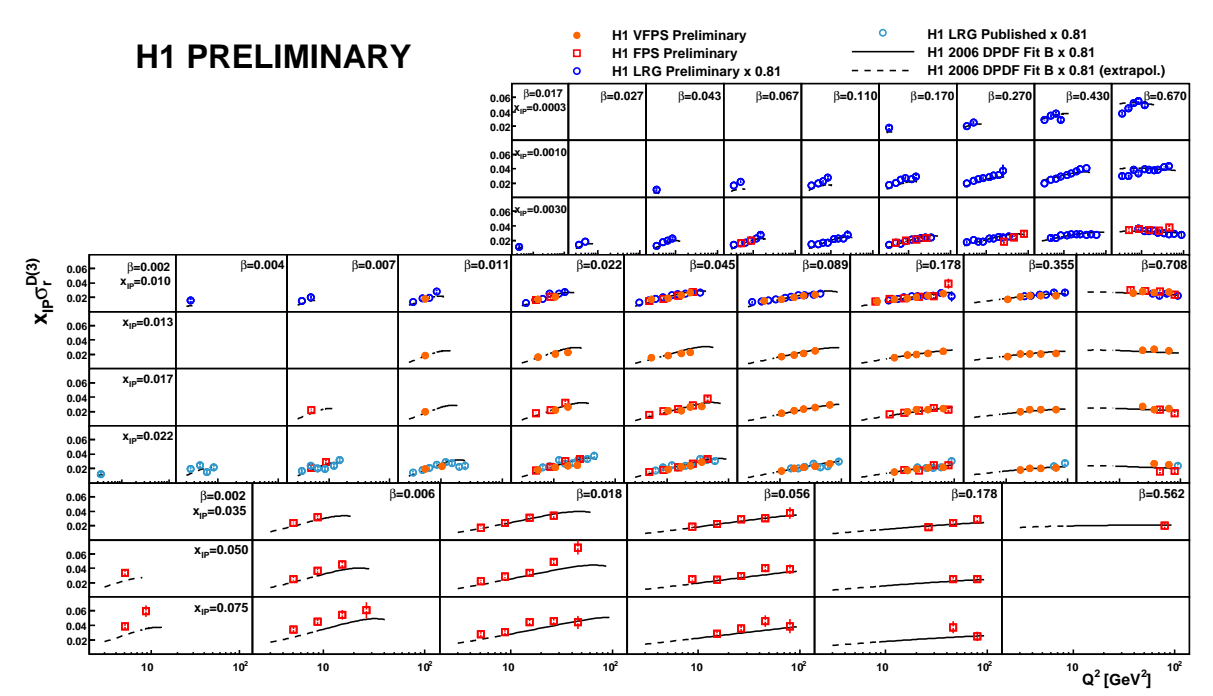

Figure 7: Diffractive reduced cross section measured by $\mathrm{H} 1$ from events wit ha large rapidity gap or with a tagged proton.

state proton staying intact or quasi-intact and by the presence of a gap in rapidity, with no activity in the detector, which separate the hadronic system from the scattered proton. In $p \bar{p}$ collisions, the observation of diffractive events is complicated by soft interactions from possible re-scattering of final state protons, which will destroy rapidity gaps. HERA therefore remains the unique place to study the exact nature of the diffractive exchange and its parton composition. Diffractive scattering can be identified via the rapidity gap or by tagging the non-dissociated proton in forward proton spectrometers. New measurements of the inclusive diffractive DIS ep $\rightarrow e X p$ cross section have been released recently by H1 [28, 29, 30] and ZEUS [31] Collaborations. As demonstrated in figure 7 summarising $\mathrm{H} 1$ measurements, they provide now a large kinematic coverage with a greatly improved accuracy. Similarly to standard DIS, a hard scattering collinear factorisation theorem [32] predicts that the cross section for diffractive DIS factorises into a set of universal diffractive parton distribution functions (DPDFs) of the proton and process-dependent hard scattering coefficients. DPDFs can then be extracted via a fit to diffractive inclusive, and also di-jet, cross sections in DIS at HERA [33]. Such DPDFs are used for example at the Tevatron to predict the background arising from inclusive diffraction in the exclusive diffractive production of heavy objects as di-jets [34]. DPDFs extracted from HERA data will therefore be an important input to the forward physics program at the LHC, in particular for the search of central exclusive Higgs production [35].

\section{References}

[1] J. C. Collins, D. E. Soper and G. F. Sterman, Nucl. Phys. B 261 (1985) 104.

[2] V. N. Gribov and L. N. Lipatov, Sov. J. Nucl. Phys. 15 (1972) 438; L. N. Lipatov, Sov. J. Nucl. Phys. 20 (1975) 94; Y. L. Dokshitzer, Sov. Phys. JETP 46 (1977) 641; G. Altarelli and G. Parisi, Nucl. Phys. B 126 (1977) 298.

[3] G. Watt, available at http://projects.hepforge.org/mstwpdf/pdf4lhc/

[4] F. D. Aaron et al. [H1 and ZEUS Collaborations], JHEP 1001 (2010) 109 [arXiv:0911.0884]. 
[5] H1 and ZEUS Collaborations, H1prelim-10-141, ZEUS-prel-10-017, July 2010.

[6] H1 and ZEUS Collaborations, H1prelim-10-142, ZEUS-prel-10-018, July 2010.

[7] F. D. Aaron et al. [H1 Collaboration], Eur. Phys. J. C 67 (2010) 1 [arXiv:0911.5678].

[8] F. D. Aaron et al. [H1 Collaboration], Eur. Phys. J. C 65 (2010) 363 [arXiv:0904.3870].

[9] ZEUS Collaboration, ZEUS-prel-10-002, April 2010.

[10] ZEUS Collaboration, ZEUS-prel-10-003, April 2010.

[11] ZEUS Collaboration, ZEUS-prel-10-014, July 2010.

[12] T. Aaltonen et al. [CDF Collaboration], Phys. Rev. D 78 (2008) 052006 [Erratum-ibid. D 79 (2009) 119902] [arXiv:0807.2204].

[13] V. M. Abazov et al. [D0 Collaboration], Phys. Rev. Lett. 101 (2008) 062001 [arXiv:0802.2400].

[14] E. Tassi, available at https://www.desy.de/h1zeus/combined_results/benchmark/tev.html

[15] A. D. Martin et al., Eur. Phys. J. C 63 (2009) 189 [arXiv:0901.0002].

[16] H. L. Lai et al., Phys. Rev. D 82 (2010) 074024 [arXiv:1007.2241].

[17] V. M. Abazov et al. [D0 Collaboration], Phys. Rev. D 80 (2009) 111107 [arXiv:0911.2710].

[18] G. Altarelli and G. Martinelli, Phys. Lett. B 76 (1978) 89.

[19] A. M. Cooper-Sarkar et al., Z. Phys. C 39 (1988) 281.

[20] H1 and ZEUS Collaborations, H1prelim-10-043, ZEUS-prel-10-001, March 2010.

[21] H1 and ZEUS Collaborations, H1prelim-09-171, ZEUS-prel-09-015, October 2009.

[22] H1 and ZEUS Collaborations, H1prelim-10-143, ZEUS-prel-10-019, September 2010.

[23] ZEUS Collaboration, ZEUS-prel-10-004, June 2010.

[24] V. M. Abazov et al. [D0 Collaboration], Phys. Rev. Lett. 102 (2009) 192002 [arXiv:0901.0739].

[25] T. Aaltonen et al. [CDF collaboration], Phys. Rev. D 79 (2009) 052008 [arXiv:0812.4458].

[26] T. Aaltonen et al. [CDF Collaboration], Phys. Rev. Lett. 100 (2008) 091803 [arXiv:0711.2901].

[27] T. Aaltonen et al. [CDF Collaboration], Phys. Rev. Lett. 104 (2010) 131801 [arXiv:0909.1505].

[28] H1 Collaboration, H1prelim-10-011, April 2010.

[29] F. D. Aaron et al. [H1 Collaboration], arXiv:1010.1476 [hep-ex].

[30] H1 Collaboration, H1prelim-10-014, April 2010.

[31] S. Chekanov et al. [ZEUS Collaboration], Nucl. Phys. B 816 (2009) 1 [arXiv:0812.2003].

[32] J. C. Collins, Phys. Rev. D 57 (1998) 3051 [Erratum-ibid. D 61 (2000) 019902] [hep-ph/9709499].

[33] S. Chekanov et al. [ZEUS Collaboration], Nucl. Phys. B 831 (2010) 1 [arXiv:0911.4119].

[34] T. Aaltonen et al. [CDF Collaboration], Phys. Rev. D 77 (2008) 052004 [arXiv:0712.0604].

[35] S. Heinemeyer et al., Eur. Phys. J. C 53 (2008) 231 [arXiv:0708.3052]; J. R. Ellis, J. S. Lee and A. Pilaftsis, Phys. Rev. D 71 (2005) 075007 [hep-ph/0502251]. 